\title{
Factors Associated with Incident Cardiovascular Events and Cardiac Risk Assessment in a Cohort of HIV-infected Participants in Rio de Janeiro
}

\author{
Rodrigo Carvalho Moreira ${ }^{1,2, *}$, Mariana Alencar Miranda ${ }^{3}$, Jessica Muller ${ }^{3}$, \\ Sandra Wagner Cardoso ${ }^{2}$, Ronaldo Ismerio Moreira ${ }^{2}$, Estevao Nunes ${ }^{2}$, Rosane Griep ${ }^{4}$, \\ Maria Jesus Fonseca ${ }^{5}$, Valdilea Veloso ${ }^{2}$, Beatriz Grinsztejn ${ }^{2}$, Dora Chor ${ }^{5}$, \\ Antonio Guilherme Pacheco ${ }^{1}$ \\ ${ }^{1}$ Oswaldo Cruz Foundation, Scientific Computing Program, Rio de Janeiro, Brazil \\ ${ }^{2}$ Oswaldo Cruz Foundation, National Institute of Infectology Evandro Chagas, Rio de Janeiro, Brazil \\ ${ }^{3}$ Internal Medicine, Universidade Federal do Rio de Janeiro, Rio de Janeiro, Brazil \\ ${ }^{4}$ Oswaldo Cruz Foundation, Laboratory of Health and Environment Education, Rio de Janeiro, Brazil \\ ${ }^{5}$ Oswaldo Cruz Foundation, Department of Epidemiology and Quantitative Methods in Health, Rio de Janeiro, Brazil
}

\section{Email address:}

rodrigo.moreira@ini.fiocruz.br (R. C. Moreira),mari.alencarmiranda@gmail.com (M. A. Miranda), jessica_mullerdefaria@hotmail.com(J. Muller), sandra.wagner@ini.fiocruz.br (S. W. Cardoso),

ronaldo.ismerio@ini.fiocruz.br (R. I. Moreira), estevao.nunes@ini.fiocruz.br (E. Nunes)rohgriep@gmail.com (R. Griep), mariafonseca818@gmail.com (M. J. Fonseca), valdilea.veloso@gmail.com (V. Veloso), beatriz.grinsztejn@gmail.com (B. Grinsztejn), dorachor@gmail.com (D. Chor), antgui@gmail.com (A. G. Pacheco)

${ }^{*}$ Corresponding author

\section{To cite this article:}

Rodrigo Carvalho Moreira, Mariana Alencar Miranda, Jessica Muller, Sandra Wagner Cardoso, Ronaldo Ismerio Moreira, Estevao Nunes, Rosane Griep, Maria Jesus Fonseca, Valdilea Veloso, Beatriz Grinsztejn, Dora Chor, Antonio Guilherme Pacheco. Factors Associated with Incident Cardiovascular Events and Cardiac Risk Assessment in a Cohort of HIV-infected Participants in Rio de Janeiro. International Journal of Infectious Diseases and Therapy. Vol. 6, No. 1, 2021, pp. 17-26. doi: 10.11648/j.ijidt.20210601.13

Received: January 22, 2021; Accepted: January 30, 2021; Published: February 9, 2021

\begin{abstract}
Background: Cardiovascular events (CVEs) have emerged as an important cause of morbimortality in people living with HIV/AIDS (PLHA). We aimed to investigate factors associated with incident CVE and compare the observed incidence with the predicted CVE risk by three different equations. Methods: Among the 649 active participants of the INIELSA cohort, we analysed data of 644 individuals free of CVE at baseline examinations. We used Cox regression models to study factors associated with CVE and to assess the equation's performance we compared the observed incidence with the overall 5-year predicted risks. Results: Over a median follow-up of 6.26-years (interquartile range [IQR] 6.01-6.47), there were 18 CVE, four deaths. Overall, $57.92 \%$ were male, median age was 43.4 years (35.82-50.73), most were on cART (88.65\%). Participants who had CVE were older, had poorer renal function, higher proportion of dyslipidemia and higher levels of systolic blood pressure and triglycerides. The observed CVE rate was $2.90 \%(1.60-4.90 \%)$ whereas their overall cardiovascular disease (CVD) risk differed depending on which score was used: $0.91(0.42-20), 1.16(0.38-2.78)$ and $1.68(0.60-3.87)$, according to Framingham, ASCVD and DAD, respectively. In the multivariable Cox analysis black race, smoking 10+ packs years, dyslipidemia, current CD4 cell count $\times 10^{1}$, cumulative viral load and time on NNRTI were independent predictors of CVE. Conclusion: Both traditional and HIV-related factors were associated with incident CVE. Risk scores were inconsistent in classifying patients who had CVE as high risk, indicating that validation studies are required to improve risk assessment of PLHA in Brazil.
\end{abstract}

Keywords: Acquired Immunodeficiency Syndrome, Cardiovascular Disease, Risk Assessment 


\section{Introduction}

Although in the last decades HIV infection and its related complications remained as the major causes of death in people living with HIV/AIDS (PLHA), mounting data suggest that cardiovascular disease (CVD) have emerged as important causes of morbimortality in PLHA, after the introduction of combined antiretroviral therapy (cART).

The etiology seems to be multifactorial; the main components of traditional CVD risk factors for the development of CVD are increasingly present in PLHA, as recent epidemiologic studies showed higher rates of diabetes, smoking and hypertension in PLHA when compared to the general population [1-4]. In addition, some cART regimens have been implicated in the induction of abnormal central fat accumulation, which in turn, promotes dyslipidemia and insulin resistance [5-7]. Consequently, HIV infection has been associated with $50 \%$ increase in myocardial infarction risk, even after controlling for well-known cardiovascular (CV) risk factors [8]. Although traditional risk factors do not fully explain the increased CVD risk, whether HIV infection is itself a risk factor by promoting immune activation and inflammation is not totally clear yet. Notwithstanding, it is of uppermost importance for clinicians to correctly recognize high-risk patients in order to initiate preventive interventions to control modifiable risk factors. To address this purpose, several risk functions have been derived from epidemiologic prospective studies in the general population. The most widely used in clinical practice, the Framingham risk score (FRS), was the first to demonstrate the ability to predict sex-specific 10-year CVD risk using a set of variables obtained from a Caucasian population, using a mathematical model [9]. Since then, it has been further validated for use in different populations, provided that adjustments are made for demographic variation and the underlying CVD rates [10,11]. Recently, Framingham has been adopted for PLHA, generating criticism and debate in the literature about this approach, since PLHA hold unique characteristics [12-15]. The Data Collection on Adverse Events of Anti-HIV Drugs (DAD) consortium developed a risk prediction equation specifically for PLHA, taking into account traditional and HIV-related CVD risk factors, and it was further updated to include a marker of immune function, even though its external validity is limited [16]. Lastly, the American College of Cardiology/American Heart Association (ACC/AHA) presented the Atherosclerotic Cardiovascular Disease Risk Score (ASCVD), whose difference is to include race to the algorithm, requiring further validation in external cohorts [17].

Accordingly, we previously reported that virologic suppression and higher CD4 counts were as important as traditional CVD risk factor for determining an incident cardiovascular events (CVE) among hospitalized PLHA of our cohort [18]. Nevertheless, there is scarce information in the literature about CVE incidence in PLHA who are followed in outpatient facilities in Brazil and the contribution of risk factors, either linked or not to HIV infection. This information could improve clinical practice considering its contribution to a better approach driving preventive interventions for CVD risk reduction in PLHA. Therefore, we propose to investigate factors associated with incident CVE in PLHA in Brazil and secondarily to compare the predicted 5-year risk calculated by FRS, DAD and ASVCD with the observed CVE in our cohort.

\section{Methods}

The Instituto Nacional de Infectologia Evandro Chagas (INI) is a national reference center for infectious diseases, research, and training related to HIV/AIDS since 1986. An observational clinical database, available in electronic and paper records, is maintained, with PLHA receiving primary and tertiary HIV care at its facilities, which constitutes the INI cohort. Among the active participants of the INI cohort who were between the ages of 25 and 75 in $2011(n=2,372), 649$ were randomly selected and invited to the INI-ELSA study, ELSA coming from the Brazilian Longitudinal Study of Adult Health (ELSA-Brasil)[19]. Among those, 644 free of CVD at baseline visit were included in this study and prospectively followed until Dec 2017 for the occurrence of CVE.

\subsection{Data Collection}

Baseline visits consisted of the same examinations of the ELSA-Brasil, which aims to contribute relevant information with respect to the development and progression of chronic diseases, particularly cardiovascular and diabetes [20-23].

\subsection{Baseline-visit}

During the accrual period, between Jan 2011 and Dec 2011, participants underwent the ELSA-Brasil protocol workup, which included detailed interviews with standard questionnaires, covering sociodemographic characteristics, use of prescription and non-prescription medications in the last month and comorbidity characteristics [20]. In addition, an extensive workup including clinical and anthropometric examinations, oral glucose tolerance test; fasting venous blood samples were collected for a complete laboratory evaluation. Besides, regular information of INI cohort participants was also obtained from medical charts in the cohort's database, including use of cART, protease inhibitor (PI), non-nucleoside reverse transcriptase inhibitors (NNRTI) and Nucleoside reverse transcriptase inhibitors (NRTI)-containing regimens and the length in years on each regimen, current CD4 counts, viral loads, cytomegalovirus (CMV) serology, nadir CD4 counts and previous HIV viral load measurements.

\subsection{Follow-up Visits}

Clinical events, whether or not associated with HIV/AIDS, antiretroviral treatment regimens and adverse events were systematically collected retrospectively from medical records. This work was carried out by a team of specialized and trained technicians, under the supervision of an 
infectologist. HIV viral loads and CD4 + cell counts were measured every 4-6 months as part of regular outpatient care. Lymphocyte CD4 count was performed by flow cytometry (Becton-Dickinson, USA) and HIV viral load quantification is measured by the NUCLISENS methodology, with a minimum detection limit of 40 copies $/ \mathrm{ml}$.

\subsection{Outcome Definition}

For the purposes of this study a CVE was defined as a composite of incident acute myocardial infarction (AMI), angina, stroke, heart failure or peripheral artery disease (PAD). In order to improve the quality of information, a systematic validation of patients who had CVEs recorded in their chart was performed by a cardiologist according to current guidelines [24-29]. Cardiovascular deaths that occurred in the study period were also monitored retrieving data from the coding causes of death in HIV (CoDe) system (http://www.cphiv.dk/DAD/). For the analysis, when a participant experienced an event of interest he was no more considered at risk.

\subsection{Covariables Definitions}

Variables were defined or calculated as follows. Self-reported race/skin color was categorized into black, brown, white, and others. Diabetes mellitus (DM) was defined as having glycated hemoglobin $>6.5 \%$, fasting glucose $>126 \mathrm{mg} / \mathrm{dL}$, oral glucose tolerance test levels $>200 \mathrm{mg} / \mathrm{dL}$ or current use of a hypoglycemic drug. Resting blood pressure was measured three times in the non-dominant arm in the sitting position and hypertension was ascertained if the mean of the last 2 measurements showed systolic blood pressure $\geq 140 \mathrm{mmHg}$ or diastolic blood pressure $\geq 90 \mathrm{mmHg}$ or if in use of antihypertensive drugs. Dyslipidemia was defined as low-density lipoprotein (LDL) levels $\geq 130 \mathrm{mg} / \mathrm{dL}$ or use of lipid-lowering drugs. Cumulative smoking was calculated as pack-years, by multiplying the number of packs smoked per day by the number of years of smoking. Family history of sudden death and family history of acute myocardial infarction (AMI) or stroke were defined as the informed occurrence of any episode of those conditions among parents or siblings younger than 65 years of age. Body mass index (BMI) was calculated as the ratio of measured weight in kilograms and squared height in meters. Cumulative viral load (VL; viremia copy-years) was defined as the area under the curve for two consecutive viral load measurements over time, in years [30]. Cumulative use of antiretroviral therapy was calculated as the sum of the difference between start and end dates of use up to the follow-up termination, in years. Estimated Glomerular filtration rate (eGFR) was calculated with the CKD-Epi equation.

\subsection{CVD Risk Equations}

The predicted 5-year CVD risk was calculated for each participant at baseline using three CVD risk equations: 1) the FRS risk equation, calculated as a combination of age, sex, systolic blood pressure (SBP), antihypertensive therapy, serum total cholesterol (TC) and HDL-cholesterol (HDL-c) values, current smoking status and diabetes. 2) The DAD CVD equation estimates risk by combining information for age; sex; SBP; serum TC and HDL-c, diabetes; smoking status; family history of CVD; current use of abacavir, indinavir, or lopinavir; and the number of years on indinavir or Lopinavir. 3) The ASCVD risk was estimated using age, diabetes, sex, race, smoking status, SBP, treatment for hypertension, TC and HDL-c. Then, for comparison proposes the 5-year risk of coronary heart disease was arbitrarily categorized as low $(<1 \%)$, moderate ( 1 to $5 \%)$ and high $(>5 \%)$ for each equation.

\section{Statistical Analysis}

Descriptive comparisons of general characteristics between groups, including predicted and observed CVE were done with Mann-Whitney tests for continuous and chi-squared or Fisher exact tests for categorical variables. Data were expressed as median [interquartile range](IQR) or frequency (\%) where applicable. To study factors associated with CVE in PLHA, the best multivariable model among a set of candidate variables was chosen for Cox proportional hazards regression using a procedure implemented by a genetic algorithm, useful when we have a large candidate sets, and ranked by the Akaike information criterion. The procedure was also replicated 20 times and the overall best model was chosen through exhaustive screening. To assess the equations' performances, we compared the observed number of CVE with the absolute 5-year risk calculated by FRS, DAD and ASVCD. The observed number was adjusted for probability of event-free survival using the Kaplan-Meier (KM) estimator based on a tabulation of the number of PLHA at risk and the number of CVE at each unique time. For the times in which an event occurred, the probability of survival was estimated by the number of those who survived until that time over those who were at risk. The survival function estimated was the product of the probability of survival at each time and the adjusted observed number was the complement of that function. P-values less than 5\% considered significant for differences between groups. All analyses were performed in the $\mathrm{R}$ environment version 3.2.2 [31].

\section{Results}

Among the 644 participants, followed for a median of 6.26years (interquartile range [IQR] 6.01-6.47), there were $18 \mathrm{CVE}$ : $6(33.33 \%)$ acute myocardial infarctions, 2 (11.11\%) strokes, 6 $(33.33 \%)$ unstable anginas and $4(22.23 \%)$ cardiovascular deaths. The event rate was 4.46/1000 person-years (4031 person-years of follow-up), yielding a cumulative five-year survival rate of $97.1 \%(95 \% \mathrm{CI}=95.8-98.4)$. Overall, $57.92 \%$ were male, predominantly brown (40.53\%), and the median age was 43.4 (35.82-50.73) years. Among traditional CV risk factors, smoking was found in $23.45 \%$, dyslipidemia in $37.24 \%$, hypertension in $31.06 \%$ and diabetes in $25.47 \%$. Participants who suffered a CVE were older (median age 51.66 years), had higher levels of triglycerides and dyslipidemia, with a decreased glomerular filtration rate estimated by CKD-Epi (median 91.52 $\mathrm{mL} / \mathrm{min}$ per $1.73 \mathrm{~m}^{2}$ ). The main clinical and laboratory 
characteristics of the study participants with or without CVE are shown in Table 1. Regarding HIV-related characteristics, at baseline most of them were under cART $(88.65 \%)$ for a median time of 9.82 years $(6.3-16.32)$ with undetectable viral load (71.27\%) and high levels of current CD4 cell counts (median 540 [IQR=371-739.25]) and the most commonly used regimen was NRTI (81.68\%). The median of current lower CD4 cell count at baseline visit was significantly lower and the cumulative viral load was higher in participants who had a CVE, when compared to those who did not; cART regimens and HIVrelated characteristics of PLHA are depicted in Table 2.

Table 1. General characteristics of PLHA according to the incidence of cardiovascular event.

\begin{tabular}{|c|c|c|c|c|}
\hline & \multicolumn{4}{|l|}{ Groups } \\
\hline & CVE- & CVE+ & Total & p value $^{b}$ \\
\hline Total & 626 & 18 & 644 & \\
\hline Sex: Male & $361(57.67)$ & $12(66.67)$ & $373(57.92)$ & 0.4458 \\
\hline Age in years - median (IQR) & $43.13(35.8,50.16)$ & $51.66(47.66,53.84)$ & $43.4(35.82,50.73)$ & 0.0091 \\
\hline Schooling: $<9$ years & $296(47.28)$ & $10(55.56)$ & $306(47.52)$ & 0.4884 \\
\hline Race & & & & 0.7105 \\
\hline Brown & $254(40.58)$ & $7(38.89)$ & $261(40.53)$ & \\
\hline White & $205(32.75)$ & $5(27.78)$ & $210(32.61)$ & \\
\hline Others & $26(4.15)$ & $0(0)$ & $26(4.04)$ & \\
\hline Smoking & & & & 0.5448 \\
\hline Never & $316(50.48)$ & $7(38.89)$ & $323(50.16)$ & \\
\hline Former & $165(26.36)$ & $5(27.78)$ & $170(26.4)$ & \\
\hline Current & $145(23.16)$ & $6(33.33)$ & $151(23.45)$ & \\
\hline Income (R\$) - median (IQR) & $414.5(207.25,1036.25)$ & $414.5(207.25,880.81)$ & $414.5(207.25,1036.25)$ & 0.3659 \\
\hline Family History of Sudden Death & $118(18.85)$ & $2(11.11)$ & $120(18.63)$ & 0.549 \\
\hline Family History of AMI or Stroke & $173(27.64)$ & $2(11.11)$ & $175(27.17)$ & 0.1776 \\
\hline Total cholesterol (mg/dL) - median (IQR) & $181(155.25,210.75)$ & $202.5(171.25,226.75)$ & $182(156,211)$ & 0.1551 \\
\hline $\mathrm{HDL}(\mathrm{mg} / \mathrm{dL})-$ median (IQR) & $42(35,52)$ & $42(32.5,51.75)$ & $42(35,52)$ & 0.7244 \\
\hline LDL (mg/dL) - median (IQR) & $106(87,133)$ & $108(94,131)$ & $106(87,133)$ & 0.5887 \\
\hline Triglycerides (mg/dL) - median (IQR) & $119(84,181)$ & $192.5(165.75,243.75)$ & $120(85,184.5)$ & $<0.001$ \\
\hline Dyslipidemia & $224(36.48)$ & $11(64.71)$ & $235(37.24)$ & 0.0176 \\
\hline Use of lipid-lowering drugs & $81(12.94)$ & $7(38.89)$ & $88(13.66)$ & 0.0063 \\
\hline Hypertension & $191(30.51)$ & $9(50)$ & $200(31.06)$ & 0.0781 \\
\hline Antihypertensive medication use & $138(22.04)$ & $5(27.78)$ & $143(22.2)$ & 0.5675 \\
\hline $\mathrm{DM}$ & $157(25.08)$ & $7(38.89)$ & $164(25.47)$ & 0.181 \\
\hline Use of antidiabetic drugs & $32(5.11)$ & $3(16.67)$ & $35(5.43)$ & 0.0684 \\
\hline Waist/hip ratio - median (IQR) & $0.9(0.84,0.96)$ & $0.94(0.88,0.98)$ & $0.9(0.84,0.96)$ & 0.0631 \\
\hline BMI - median (IQR) & $24.45(21.99,27.37)$ & $23.6(21.39,28.18)$ & $24.43(21.93,27.51)$ & 0.8628 \\
\hline hs-CRP (mg/dL) - median (IQR) & $0.29(0.17,0.64)$ & $0.27(0.21,0.96)$ & $0.29(0.17,0.65)$ & 0.5358 \\
\hline DBP - median (IQR) & $76.75(70.5,83.38)$ & $79.5(73.5,91.62)$ & $77(70.5,83.5)$ & 0.1554 \\
\hline
\end{tabular}

${ }^{\text {a }}$ Columns provide numbers (\%) and interquartile range (IQR) where applicable

b. Mann-Whitney and Chi-Square tests

Abbreviations: IQR - interquartile range; AMI - acute myocardial infarction; CVE - cardiovascular event; BMI - body mass index; DM - diabetes mellitus; HDL - high density lipoprotein; LDL - low density lipoprotein; hs-CRP high sensitivity C-reactive protein; CKD-Epi - Chronic Kidney Disease Epidemiology Collaboration equation; SBP - systolic blood pressure; DBP - diastolic blood pressure

Multivariable Cox regression analysis showed that selfreported black race/color (hazard ratio $[\mathrm{HR}]=7.96$, $95 \% \mathrm{CI}=1.74 ; 36.48, \mathrm{p}=0.007)$, smoking $10+$ packs years $(\mathrm{HR}=4.05, \quad 95 \% \mathrm{CI}=1.22 ; \quad 13.42, \quad \mathrm{p}=0.02), \quad$ dyslipidemia $(\mathrm{HR}=6.09,95 \% \mathrm{CI}=1.85 ; 19.98, \mathrm{p}<0.01)$, cumulative viral load $(\mathrm{HR}=1.06,95 \% \mathrm{CI}=1.02 ; 1.10, \mathrm{p}<0.01)$ and time on NNRTI $\quad(\mathrm{HR}=1.24,95 \% \mathrm{CI}=1.07 ; 1.48, \quad \mathrm{p}<0.01)$, were independent predictors of increased CVE whereas time on NRTI in years $(\mathrm{HR}=0.77,95 \% \mathrm{CI}=0.65 ; 0.91, \mathrm{p}<0.01)$ and every 10 units increase in current CD4 cell count $(\mathrm{HR}=9.97$, $95 \% \mathrm{CI}=9.94 ; 9.99, \mathrm{p}=0.01$ ) had a protective effect (Table 3 ).

Estimated CVD risk equations

The estimated 5-year interquartile range risk varied between $0.6 \%$ and $3.87 \%$, with a median of $1.68 \%$ based on the DAD equation, from 0.42 to $2 \%$ with a median of $0.91 \%$ based on the FRS and from 0.38 to $2.78 \%$ with a median of $1.16 \%$ based on the ASCVD calculator, whereas the observed 5-year KM adjusted CVE was 2.90\% (95\% CI=1.6-4.90\%) (Figure 1). Overall, the categorized 5-year risk showed that DAD, FRS and ASCVD equations estimated $17.46 \%, 4.84 \%$ and $13.73 \%$ of the study population to be at high risk, respectively. Among those who had a CVE, the DAD equation had higher 5-year median values (4.05 $[\mathrm{IQR}=2.33-5.6])$, when compared to FRS (2.43 $[\mathrm{IQR}=1.32-3.31])$ and to ASCVD calculator $(2.73[\mathrm{IQR}=1.49$ 5.35]), and both DAD and ASVCD classified a larger proportion as high risk (33.33\%) than FRS (5.56\%). The overall absolute 5year CVE risk estimates and the risk categories of DAD, FRS and ASVCD equations in PLHA are exhibited in Table 4. 
Table 2. cART regimens and HIV-related characteristics of PLHA by incidence of CVE.

\begin{tabular}{|c|c|c|c|c|}
\hline & CVE- & CVE+ & Total & p-value ${ }^{b}$ \\
\hline Total & 626 & 18 & 644 & \\
\hline cART use: Yes & $554(88.64)$ & $16(88.89)$ & $570(88.65)$ & 0.99 \\
\hline PI-containing regimen & $326(52.08)$ & $11(61.11)$ & $337(52.33)$ & 0.4493 \\
\hline NNRTI-containing regimen & $407(65.02)$ & $12(66.67)$ & $419(65.06)$ & 0.8849 \\
\hline NRTI-containing regimen & $510(81.47)$ & $16(88.89)$ & $526(81.68)$ & 0.5502 \\
\hline Cumulative Viral Load-median (IQR) & $15.12(6.88,29.46)$ & $31.58(9.66,42.02)$ & $15.28(7,29.84)$ & 0.0492 \\
\hline Current CD4 counts (cells $/ \mathrm{mL}$ )—median (IQR) & $540.5(374,740.75)$ & $407.5(230,595.75)$ & $540(371,739.25)$ & 0.0404 \\
\hline Undetectable Viral Load: Yes & $441(71.24)$ & $13(72.22)$ & $454(71.27)$ & 0.928 \\
\hline Time on cART (years)-median (IQR) & $9.78(6.27,16.27)$ & $11.05(7.16,16.08)$ & $9.82(6.3,16.32)$ & 0.6297 \\
\hline Time on PI (years) - median (IQR) & $4.66(0,11.15)$ & $9.03(0.99,13.12)$ & $4.69(0,11.26)$ & 0.2482 \\
\hline Time on NNRTI (years)—median (IQR) & $3.18(0.08,6.8)$ & $2.54(0,8.69)$ & $3.18(0.07,6.84)$ & 0.9421 \\
\hline Time on NRTI (years) - median (IQR) & $8.88(5.79,15.96)$ & $10.08(6.38,14.37)$ & $8.88(5.8,15.95)$ & 0.9326 \\
\hline
\end{tabular}

${ }^{a}$ Columns provide numbers (\%) and interquartile range (IQR) where applicable

b. Mann-Whitney and Chi-Square tests

Abbreviations: cART — combined antiretroviral therapy; PI - protease inhibitors; NNRTI - Non-nucleoside reverse transcriptase inhibitors, NRTI Nucleoside reverse transcriptase inhibitors

Table 3. Final Cox model for clinical predictors of CVD in in people living with HIV/AIDS.

\begin{tabular}{llll}
\hline Variable & HR & 95\%IC & p-value \\
\hline Sex: Male & 0.858 & $0.274 ; 2.686$ & 0.7929 \\
Age in years & 0,999 & $0.935 ; 1.067$ & \\
Race & & & \\
Black & 7.962 & $1.737 ; 36.485$ & 0.9822 \\
Brown & 2.360 & $0.540 ; 10.303$ & 0.0076 \\
Pack. years (10+) & 4.053 & $1.224 ; 13.420$ & 0.2534 \\
Family History of AMI or Stroke & 0.137 & $0.023 ; 0.792$ & 0.0220 \\
Dyslipidemia & 6.086 & $1.853 ; 19.985$ & 0.0264 \\
Diabetes Mellitus & 2.773 & $9.94 ; 9.99$ & 0.0029 \\
Current CD4+ cell count $\left(10^{1}\right)$ & 9.97 & $0.954 ; 1.048$ & $0.932 ; 0.982$ \\
HDL (mg/dL) & 1.001 & $1.023 ; 1.101$ & 0.0658 \\
CKD-Epi & 0.957 & $1.070 ; 1.476$ & 0.9857 \\
Cumulative Viral Load & 1.062 & $0.650 ; 0.913$ \\
Time on NNRTI (years) & 1.257 & $0.985 ; 1.292$ \\
Time on NRTI (years) & 0.770 & 0.0009 \\
Time on IP (years) & 1.128 & 0.0013 \\
\hline
\end{tabular}

Abbreviations: CVE - cardiovascular events; DM - diabetes mellitus; cART — combined antiretroviral therapy; PI-protease inhibitors; NNRTI- Nonnucleoside reverse transcriptase inhibitors, NRTI - Nucleoside reverse transcriptase inhibitors

Table 4. Absolute CVD risk scores and categories of DAD, FRS and ASVCD equations in people living with HIV/AIDS.

\begin{tabular}{|c|c|c|c|c|}
\hline & \multicolumn{4}{|l|}{ Groups } \\
\hline & CVE- & CVE+ & Total & p value $^{b}$ \\
\hline Total & 610 & 11 & 621 & \\
\hline DAD 5 years median (IQR) & $1.6(0.58,3.69)$ & $4.05(2.33,5.6)$ & $1.68(0.6,3.87)$ & $<0.001$ \\
\hline DAD 5 years categorized & & & & \\
\hline High & $104(16.99)$ & $6(33.33)$ & $110(17.46)$ & 0.0302 \\
\hline Moderate & $264(43.14)$ & $10(55.56)$ & $274(43.49)$ & \\
\hline Low & $244(39.87)$ & $2(11.11)$ & $246(39.05)$ & \\
\hline FRS 5 years categorized & & & & 0.0076 \\
\hline High & $30(4.82)$ & $1(5.56)$ & $31(4.84)$ & \\
\hline Moderate & $261(41.89)$ & $14(77.78)$ & $275(42.9)$ & \\
\hline Low & $332(53.29)$ & $3(16.67)$ & $335(52.26)$ & \\
\hline ASVCD 5 years median (IQR) & $1.14(0.35,2.69)$ & $2.73(1.49,5.35)$ & $1.16(0.38,2.78)$ & 0.0024 \\
\hline ASCVD 5 years categorized & & & & 0.0049 \\
\hline High & $82(13.16)$ & $6(33.33)$ & $88(13.73)$ & \\
\hline Low & $283(45.43)$ & $2(11.11)$ & $285(44.46)$ & \\
\hline
\end{tabular}

a Abbreviations: Framingham Risk Score (FRS), Data Collection on Adverse Events of Anti-HIV Drugs (D: A: D) and Atherosclerotic Cardiovascular Disease

Risk Score (ASCVD); Columns provide numbers (\%) and interquartile range (IQR) where applicable

b. Mann-Whitney and Chi-Square tests 


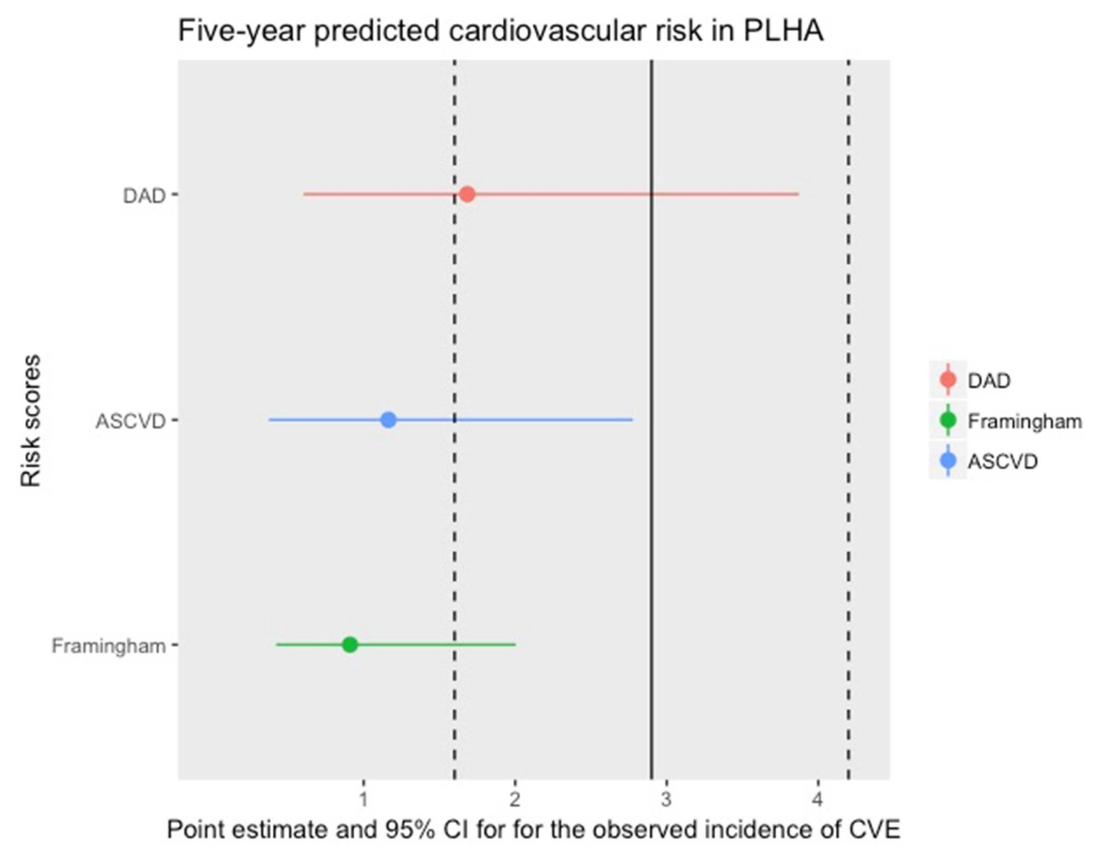

Figure 1. The five year predicted cardiovascular risk in people living with HIV/AIDS.

The $\mathrm{x}$-axis shows the point estimate (solid line) and 95\% confidence interval (dashed lines) for the observed incidence of cardiovascular events (CVE). In the Y-axis are the predicted probabilities (median and interquartile range) for The Data Collection on Adverse Events of Anti-HIV Drugs (DAD), Atherosclerotic Cardiovascular Disease (ASCVD) and Framingham risk score.

\section{Discussion}

In this study we showed that traditional $\mathrm{CV}$ risk factors and HIV-specific characteristics were independent predictors of CVE in PLHA, whereas higher CD4 counts were cardioprotective. Moreover, the DAD equation seems to better predict CVE in a Brazilian cohort of PLHA, compared to other two widely used ones.

Our main findings are in agreement with several studies. For instance, De Socio et al recently found age, smoking and diabetes as predictors of CVE in PLHA [32]. For Quiros et al., in a population-based study of PLHA in northern Italy, age greater than 45 years, male gender, diabetes, and total cholesterol were significantly associated with CVE [33].

It is noteworthy that he magnitude of effect for black race was substantial as a predictor of CVE. Aside from genetic and environmental influences, lifetime risk of CVE in the general population is dependent on the burden of risk factors jointly present. Thereupon, epidemiological studies reveal higher prevalences of hypertension, diabetes and obesity in African Americans than whites [34-36]. In the Brazilian setting, participants of black race from the ELSA-Brasil study had poorer metrics of cardiovascular related health and lifestyle factors, along with early detection of atherosclerosis but it was not possible to establish an association between racial discrimination and hypertension [37-39]. Furthermore, there has been evidence of racial disparities in cardiovascular health care utilization and treatments that would lead to adverse clinical outcomes [40, 41]. Altogether, it might be that the same mechanisms that cause blacks to have a higher occurrence of cardiovascular disease are operating in people living with HIV as well.

We also detected a strong association between smoking $10+$ packs years and CVE. Phan B et all found similar results, where baseline carotid plaque was independently associated with pack per year of smoking [42] and for Hsue PY et all, cigarette pack-years was a predictor of intimamedia thickness [43]. These results, coupled with epidemiologic studies that show rates of smoking two to three times higher among PLHA than age-matched controls $[44,45]$, reinforce the importance of strategies to stop smoking and its gains in primary prevention.

The HIV-induced proinflammatory state may contribute to the overall increased number of CVE in PLHA, particularly in those not on ART [46]. In this scenario of cART use our results showed that higher levels of current CD4 was cardioprotective and in the unadjusted analysis we observed that parameters of immune activation and viral activity such as current CD4 cell count and undetectable viral load were in optimal conditions as a consequence of HIV treatment. We suppose that the large proportion of cART use $(88.39 \%)$ in our cohort had a relevant role in inducing control of chronic inflammation and vascular damage as potential pathogenic mechanisms, promoting an indirect effect in CVE rate reduction. This hypothesis is corroborated by data from SMART and ESPRIT randomized clinical trials, whose cART arms included patients with well-controlled viral loads and high CD4 counts, and showed mortality rates comparable to the general population. In these studies, the most frequent cause of death was CVD [47].

We also found that viremia copy-years or cumulative viral 
load was associated with increased CVD. The cumulative viral load means a person had a detectable viral load for progressively longer times, inducing chronic inflammation. Epidemiologic studies suggest that keeping viral load consistently undetectable with cART reduces the risk of a cardiovascular event $[48,49]$. For instance, in the SMART study, cART interruption was associated with increased CVD, mainly as consequence of lowering CD4+ cell count and increasing viral load [50].

Collectively these data suggest a tangible benefit of continuous use of cART having virologic control as the underlying goal. Herein, it is fundamental the implementation of regular monitoring of comorbidities related to CVD as well as assessment of interventions and lifestyle modification following the current guidelines as part of the HIV care continuum, to further lower CV risk.

Regarding the performance of the absolute risk estimated by the three equations in our exploratory analysis, although all of them underestimated the observed risk, the DAD score performed better $(1.44 \%)$ than FRS $(0.88 \%)$ and ASVCD (1.13\%) when we compared these point estimates with the observed 5-year CVE (1.80\%). It is also important to notice that the three predicted risks are within the confidence interval width estimated for this population sample (Figure 1), but only the DAD score range included the point estimate of the observed risk. We found discordant results in the literature when assessing CVD prediction in PLHA; for instance, Thompson et al. found that DAD and ASVCD underestimated the CV risk whereas FRS accurately predicted it [51]. In accordance, De Socio observed that CVE were well predicted by FRS [32], whereas for Raggi et al., both ASVCD and FRS estimates for 10 years and DAD estimates for 5 years, have similar risks of CVE and the author concluded that all equations showed modest predictive ability [52]. More recently, Crane et al reported in a conference abstract that ASCVD had better discrimination than other 3 risk scores, including the DAD and FRS for myocardial infarction in PLHA [53]. The reasons for these conflicting results could be partially explained by the use of surrogate endpoints to measure CVE and differences in relation to demographic characteristics and CVE definition or its ascertainment among the populations in these studies.

Our results suggest that FRS could be underestimating CVE risk in PLHA in Brazil, as the predicted 5-year absolute CVD risk was the lowest amongst the three equations that have been evaluated and models that take into account exposure to individual cART regimens and immune activation could more accurately predict CVE occurrence. However, to better understand these inconsistences, formal validation of these models are necessary to determine whether one of these tools can be generalized among PLHA in Brazil.

Moreover, when we consider the absolute 5-year risk calculated by the updated DAD equation in the original cohort and the calculated risk by DAD equation in our cohort there is a considerable difference on the predicted risks $(2.49 \%$ vs.
$1.44 \%$ ), highlighting the baseline demographic imbalances between these populations.

The limitations of this study include the selection of a random sample from the INI Cohort, instead of using data for all the participants, due to logistic and budgetary limitations. Even though a specialist and a training doctor did the classification of CVE blinded to exposure status, it is possible that some degree of nondifferencial CVE misclassification could have occurred; moreover, we did not search our database for individuals who were considered CVE-free, but actually had the outcome, which could have changed our estimates towards the null hypothesis. Another limitation is the small number of CVE, decreasing the precision of the results. Although we have calculated the ASCVD including patients who were using statins the score was originally derived to guide statins initiation and therefore we could assume that the risk in this participants taking statins was even lower. The strengths of the study include a well-defined cohort along with a complete high-quality dataset of covariables and no losses of follow up, reducing the role of potential confounders, increasing internal validity. Furthermore, we independently validated the CVE classification, improving accuracy of CVE diagnosis obtained from our medical charts.

To the best of our knowledge, this is the first study to investigate incidence and factors associated with CVE, assessing its risk by three different equations in a middleincome country.

\section{Conclusion}

Traditional modifiable CV risk factors, including smoking $10+$ packs year, dyslipidemia and renal function are still major independent predictors of CVE in PLHA, and strategies towards controlling those risk factors along with smoking cessation are essential for primary prevention. It is also critical to implement public policies to mitigate health inequalities among Blacks. Continuous cART use should be reinforced in order to achieve viral suppression and consequently CVE reduction. The DAD equation seems to better predict CVE in PLHA than FRS and ASCVD; however, future studies should perform a formal validation of these tools in Brazil.

\section{Data Availability}

Due to legal and ethical restrictions prohibiting public sharing of data, only a portion of what was used in order to replicate all study findings and the supporting conclusions, will be available upon request.

\section{Conflict of Interest}

The authors have no conflict of interest to declare.

\section{Funding}

This study was funded by Fundação Carlos Chagas Filho 
de Amparo a Pesquisa do Estado do Rio de Janeiro [E26/102398/2009 to DC and E-26/201.471/ 2014 to AP]; Conselho Nacional de Desenvolvimento Científico e Tecnológico [grant numbers $0106 \quad 0010.00 \quad \mathrm{RS}, 0106$ 0212.00 BA, 01060300.00 ES, 01060278.00 MG, 0106 0115.00 SP, $01060071.00 \mathrm{RJ}, 407446 / 2012-5$ to AP and 304614/2012-2 to AP]; Programa Estratégico de Apoio à Pesquisa em Saúde [407446/2012-5 to AP].

\section{References}

[1] K. So-Armah et al., "HIV and cardiovascular disease," The lancet. HIV, vol. 7, no. 4, pp. e279-e293, Apr 2020, doi: $10.1016 /$ S2352-3018(20)30036-9.

[2] S. De Wit et al., "Incidence and risk factors for new-onset diabetes in HIV-infected patients: the Data Collection on Adverse Events of Anti-HIV Drugs (D: A: D) study," Diabetes care, vol. 31, no. 6, pp. 1224-9, Jun 2008, doi: $10.2337 / \mathrm{dc} 07-2013$.

[3] L. Galli et al., "Risk of type 2 diabetes among HIV-infected and healthy subjects in Italy," European journal of epidemiology, vol. 27, no. 8, pp. 657-65, Aug 2012, doi: 10.1007/s10654-012-9707-5.

[4] D. Klein, L. B. Hurley, C. P. Quesenberry, Jr., and S. Sidney, "Do protease inhibitors increase the risk for coronary heart disease in patients with HIV-1 infection?," Journal of acquired immune deficiency syndromes, vol. 30, no. 5, pp. 471-7, Aug 15 2002, doi: 10.1097/00126334-20020815000002 .

[5] S. A. Riddler et al., "Impact of HIV infection and HAART on serum lipids in men," JAMA : the journal of the American Medical Association, vol. 289, no. 22, pp. 2978-82, Jun 11 2003, doi: 10.1001/jama.289.22.2978.

[6] G. M. Behrens, "Cardiovascular risk and body-fat abnormalities in HIV-infected adults," The New England journal of medicine, vol. 352, no. 16, pp. 1721-2; author reply 1721-2, Apr 21 2005, doi: 10.1056/NEJM200504213521620.

[7] A. Pawlos, M. Broncel, E. Wlazlowska, E. Jablonowska, and P. Gorzelak-Pabis, "Cardiovascular risk and response to lipid lowering therapy in patients with HIV infection according to different recommendations," PloS one, vol. 15, no. 12, p. e0244675, 2020, doi: 10.1371/journal.pone.0244675.

[8] M. S. Freiberg et al., "HIV infection and the risk of acute myocardial infarction," JAMA internal medicine, vol. 173, no. 8, pp. 614-22, Apr 22 2013, doi: 10.1001/jamainternmed.2013.3728.

[9] R. B. D'Agostino, Sr. et al., "General cardiovascular risk profile for use in primary care: the Framingham Heart Study," Circulation, vol. 117, no. 6, pp. 743-53, Feb 12 2008, doi: 10.1161/CIRCULATIONAHA.107.699579.

[10] R. B. D'Agostino, Sr., S. Grundy, L. M. Sullivan, P. Wilson, and C. H. D. R. P. Group, "Validation of the Framingham coronary heart disease prediction scores: results of a multiple ethnic groups investigation," JAMA : the journal of the American Medical Association, vol. 286, no. 2, pp. 180-7, Jul 11 2001, doi: 10.1001/jama.286.2.180.

[11] J. Marrugat et al., "An adaptation of the Framingham coronary heart disease risk function to European Mediterranean areas," Journal of epidemiology and community health, vol. 57, no. 8, pp. 634-8, Aug 2003, doi: 10.1136/jech.57.8.634.

[12] S. Herrera et al., "The Framingham function overestimates the risk of ischemic heart disease in HIV-infected patients from Barcelona," HIV clinical trials, vol. 17, no. 4, pp. 131-9, Jul 2016, doi: 10.1080/15284336.2016.1177266.

[13] L. F. d. S. Pinto Neto, F. R. Dias, F. F. Bressan, and C. R. O. Santos, "Comparison of the ACC/AHA and Framingham algorithms to assess cardiovascular risk in HIV-infected patients," Brazilian Journal of Infectious Diseases, vol. 21, pp. 577-580, 2017. [Online]. Available: http://www.scielo.br/scielo.php?script=sci_arttext\&pid=S1413 $-86702017000600577 \& \mathrm{nrm}=$ iso.

[14] Y. C. Chia, H. M. Lim, and S. M. Ching, "Validation of the pooled cohort risk score in an Asian population - a retrospective cohort study," BMC cardiovascular disorders, vol. 14, p. 163, Nov 20 2014, doi: 10.1186/1471-2261-14-163.

[15] V. Estrada et al., "Risk of cardiovascular disease in patients with HIV infection undergoing antiretroviral therapy," Revista clinica espanola, vol. 220, no. 3, pp. 149-154, Apr 2020, doi: 10.1016/j.rce.2019.05.006. Riesgo de enfermedad cardiovascular en pacientes con infeccion VIH en tratamiento antirretroviral.

[16] N. Friis-Moller et al., "An updated prediction model of the global risk of cardiovascular disease in HIV-positive persons: The Data-collection on Adverse Effects of Anti-HIV Drugs (D: A: D) study," European journal of preventive cardiology, vol. 23, no. 2, pp. 214-23, Jan 2016, doi: $10.1177 / 2047487315579291$.

[17] P. Muntner et al., "Validation of the atherosclerotic cardiovascular disease Pooled Cohort risk equations," JAMA : the journal of the American Medical Association, vol. 311, no. 14, pp. 1406-15, Apr 9 2014, doi: 10.1001/jama.2014.2630.

[18] C. M. Diaz et al., "Traditional and HIV-specific risk factors for cardiovascular morbidity and mortality among HIVinfected adults in Brazil: a retrospective cohort study," BMC infectious diseases, vol. 16, p. 376, Aug 8 2016, doi: 10.1186/s12879-016-1735-4.

[19] B. Grinsztejn et al., "Changing mortality profile among HIVinfected patients in Rio de Janeiro, Brazil: shifting from AIDS to non-AIDS related conditions in the HAART era," PloS one, vol. 8, no. 4, p. e59768, 2013, doi: 10.1371/journal.pone.0059768.

[20] D. Chor et al., "[Questionnaire development in ELSA-Brasil: challenges of a multidimensional instrument]," Revista de saude publica, vol. 47 Suppl 2, pp. 27-36, Jun 2013. [Online]. Available: http://www.ncbi.nlm.nih.gov/pubmed/24346718. Questionario do ELSA-Brasil: desafi os na elaboracao de instrumento multidimensional.

[21] P. A. Lotufo, "[Setting up the longitudinal study for adult health (ELSA-Brasil]," Revista de saude publica, vol. 47 Suppl 2, pp. 3-9, Jun 2013. [Online]. Available: http://www.ncbi.nlm.nih.gov/pubmed/24346715. Construcao do Estudo Longitudinal de Saude do Adulto (ELSA-Brasil).

[22] E. M. Aquino et al., "Brazilian Longitudinal Study of Adult Health (ELSA-Brasil): objectives and design," American journal of epidemiology, vol. 175, no. 4, pp. 315-24, Feb 15 2012, doi: 10.1093/aje/kwr294. 
[23] M. I. Schmidt et al., "Cohort Profile: Longitudinal Study of Adult Health (ELSA-Brasil)," International journal of epidemiology, vol. 44, no. 1, pp. 68-75, Feb 2015, doi: 10.1093/ije/dyu027.

[24] P. T. O'Gara et al., "2013 ACCF/AHA guideline for the management of ST-elevation myocardial infarction: executive summary: a report of the American College of Cardiology Foundation/American Heart Association Task Force on Practice Guidelines: developed in collaboration with the American College of Emergency Physicians and Society for Cardiovascular Angiography and Interventions," Catheterization and cardiovascular interventions : official journal of the Society for Cardiac Angiography \& Interventions, vol. 82, no. 1, pp. E1-27, Jul 1 2013, doi: $10.1002 /$ ccd. 24776 .

[25] C. Sociedade Brasileira de, "[Guidelines for Unstable Angina and Non-ST-Segment Elevation Myocardial Infarction of the Brazilian Society of Cardiology (II Edition, 2007)]," Arquivos brasileiros de cardiologia, vol. 89, no. 4, pp. e89-131, Oct 2007, doi: 10.1590/s0066-782x2007001600015. Diretrizes (II Edicao, 2007) da Sociedade Brasileira de Cardiologia sobre angina instavel $\mathrm{e}$ infarto agudo do miocardio sem supradesnivel do segmento ST.

[26] C. P. Cannon et al., "2013 ACCF/AHA key data elements and definitions for measuring the clinical management and outcomes of patients with acute coronary syndromes and coronary artery disease: a report of the American College of Cardiology Foundation/American Heart Association Task Force on Clinical Data Standards (Writing Committee to Develop Acute Coronary Syndromes and Coronary Artery Disease Clinical Data Standards)," Circulation, vol. 127, no. 9, pp. 1052-89, Mar 5 2013, doi: 10.1161/CIR.0b013e3182831a11.

[27] A. R. Lifson et al., "Development of diagnostic criteria for serious non-AIDS events in HIV clinical trials," HIV clinical trials, vol. 11, no. 4, pp. 205-19, Jul-Aug 2010, doi: 10.1310/het1104-205.

[28] M. J. Radford et al., "ACC/AHA key data elements and definitions for measuring the clinical management and outcomes of patients with chronic heart failure: a report of the American College of Cardiology/American Heart Association Task Force on Clinical Data Standards (Writing Committee to Develop Heart Failure Clinical Data Standards): developed in collaboration with the American College of Chest Physicians and the International Society for Heart and Lung Transplantation: endorsed by the Heart Failure Society of America," Circulation, vol. 112, no. 12, pp. 1888-916, Sep 20 2005, doi: 10.1161/CIRCULATIONAHA.105.170073.

[29] J. M. Fria, R. C. Moreira. S. Wagner. R. Ismerio. S. Ribeiro. V. G. Veloso. B. Grinsztejn. A. G. Pacheco, "Validation of cardiovascular events in an HIV positive cohort HIV Evandro Chagas Institute," presented at the X Brazilian Congress of epidemiology, 2017.

[30] M. J. Mugavero et al., "Viremia copy-years predicts mortality among treatment-naive HIV-infected patients initiating antiretroviral therapy," Clinical infectious diseases : an official publication of the Infectious Diseases Society of America, vol. 53, no. 9, pp. 927-35, Nov 2011, doi: 10.1093/cid/cir526.

[31] R. C. Team. "'R: A Language and Environment for Statistical Computing."." (accessed 2018).
[32] G. V. De Socio, G. Pucci, F. Baldelli, and G. Schillaci, "Observed versus predicted cardiovascular events and allcause death in HIV infection: a longitudinal cohort study," BMC infectious diseases, vol. 17, no. 1, p. 414, Jun 12 2017, doi: 10.1186/s12879-017-2510-x.

[33] E. Quiros-Roldan et al., "Incidence of cardiovascular events in HIV-positive patients compared to general population over the last decade: a population-based study from 2000 to 2012 ," AIDS care, vol. 28, no. 12, pp. 1551-1558, Dec 2016, doi: 10.1080/09540121.2016.1198750.

[34] M. G. Buhnerkempe et al., "Prevalence of refractory hypertension in the United States from 1999 to 2014," Journal of hypertension, vol. 37, no. 9, pp. 1797-1804, Sep 2019, doi: 10.1097/HJH.0000000000002103.

[35] C. L. Jackson et al., "Black-white disparities in overweight and obesity trends by educational attainment in the United States, 1997-2008," Journal of obesity, vol. 2013, p. 140743, 2013, doi: 10.1155/2013/140743.

[36] M. C. Marshall, Jr., "Diabetes in African Americans," Postgraduate medical journal, vol. 81, no. 962, pp. 734-40, Dec 2005, doi: 10.1136/pgmj.2004.028274.

[37] I. S. Santos et al., "Carotid intima-media thickness value distributions in the Brazilian Longitudinal Study of Adult Health (ELSA-Brasil)," Atherosclerosis, vol. 237, no. 1, pp. 227-35, Nov 2014, doi: 10.1016/j.atherosclerosis.2014.09.004.

[38] L. B. M. Machado et al., "Ideal cardiovascular health score at the ELSA-Brasil baseline and its association with sociodemographic characteristics," International journal of cardiology, vol. 254, pp. 333-337, Mar 1 2018, doi: 10.1016/j.ijcard.2017.12.037.

[39] P. M. Mendes et al., "Association between perceived racial discrimination and hypertension: findings from the ELSABrasil study," Cadernos de saude publica, vol. 34, no. 2, p. e00050317, Mar 1 2018, doi: 10.1590/0102-311X00050317.

[40] R. Desai et al., "Racial and sex disparities in resource utilization and outcomes of multi-vessel percutaneous coronary interventions (a 5-year nationwide evaluation in the United States)," Cardiovascular diagnosis and therapy, vol. 9, no. 1, pp. 18-29, Feb 2019, doi: 10.21037/cdt.2018.09.02.

[41] P. Schroff, C. M. Gamboa, R. W. Durant, A. Oikeh, J. S. Richman, and M. M. Safford, "Vulnerabilities to Health Disparities and Statin Use in the REGARDS (Reasons for Geographic and Racial Differences in Stroke) Study," Journal of the American Heart Association, vol. 6, no. 9, Aug 28 2017, doi: 10.1161/JAHA.116.005449.

[42] B. A. P. Phan et al., "Utility of 2013 American College of Cardiology/American Heart Association Cholesterol Guidelines in HIV-Infected Adults With Carotid Atherosclerosis," Circulation. Cardiovascular imaging, vol. 10, no. 7, Jul 2017, doi: 10.1161/CIRCIMAGING.116.005995.

[43] P. Y. Hsue et al., "Progression of atherosclerosis as assessed by carotid intima-media thickness in patients with HIV infection," Circulation, vol. 109, no. 13, pp. 1603-8, Apr 6 2004, doi: 10.1161/01.CIR.0000124480.32233.8A.

[44] R. Mdodo et al., "Cigarette smoking prevalence among adults with HIV compared with the general adult population in the United States: cross-sectional surveys," Annals of internal medicine, vol. 162, no. 5, pp. 335-44, Mar 3 2015, doi: 10.7326/M14-0954. 
[45] J. M. Tesoriero, S. M. Gieryic, A. Carrascal, and H. E. Lavigne, "Smoking among HIV positive New Yorkers: prevalence, frequency, and opportunities for cessation," AIDS and behavior, vol. 14, no. 4, pp. 824-35, Aug 2010, doi: 10.1007/s10461-008-9449-2.

[46] C. S. S. J. A. O’Halloran, P. WG Mallon, "Dyslipidemia, Atherosclerosis and Cardiovascular Disease: An Increasingly Important Triad in an Aging Population Living With HIV," Future virology, vol. 8, no. 10, pp. 1021-1034., 2013.

[47] A. J. Rodger et al., "Mortality in well controlled HIV in the continuous antiretroviral therapy arms of the SMART and ESPRIT trials compared with the general population," Aids, vol. 27, no. 6, pp. 973-9, Mar 27 2013, doi: 10.1097/QAD.0b013e32835cae9c.

[48] R. M. Alvi et al., "Heart failure and adverse heart failure outcomes among persons living with HIV in a US tertiary medical center," American heart journal, vol. 210, pp. 39-48, Apr 2019, doi: 10.1016/j.ahj.2019.01.002.

[49] G. F. de Larranaga, A. Petroni, G. Deluchi, B. S. Alonso, and J. A. Benetucci, "Viral load and disease progression as responsible for endothelial activation and/or injury in human immunodeficiency virus-1-infected patients," Blood coagulation \& fibrinolysis : an international journal in haemostasis and thrombosis, vol. 14, no. 1, pp. 15-8, Jan 2003, doi: 10.1097/01.mbc.0000046173.06450.40.

[50] G. Strategies for Management of Antiretroviral Therapy Study et al., "CD4+ count-guided interruption of antiretroviral treatment," The New England journal of medicine, vol. 355, no. 22, pp. 2283-96, Nov 30 2006, doi: 10.1056/NEJMoa062360.

[51] A. M. Thompson-Paul et al., "Cardiovascular Disease Risk Prediction in the HIV Outpatient Study," Clinical infectious diseases : an official publication of the Infectious Diseases Society of America, vol. 63, no. 11, pp. 1508-1516, Dec 1 2016, doi: 10.1093/cid/ciw615.

[52] P. Raggi et al., "Prediction of hard cardiovascular events in HIV patients," The Journal of antimicrobial chemotherapy, vol. 71, no. 12, pp. 3515-3518, Dec 2016, doi: 10.1093/jac/dkw346.

[53] H. M. C. R. N. J. A. D. D. D. S. H. R. Y. M. J. F. R. M. M. S., "Comparing Cardiovascular Disease Risk Scores for Use in HIV-Infected Individuals. Optimal cardiovascular disease risk score for HIV " presented at the Conference on Retroviruses and Opportunistic Infections, Boston, 2016. 